\title{
ON METABELIAN TWO-KNOT GROUPS
}

\author{
JONATHAN A. HILLMAN
}

\begin{abstract}
We show that if the commutator subgroup of a 2-knot group is abelian but not finitely generated, then it is isomorphic to the additive group of dyadic rationals, thus eliminating the one possibility left open in recent work of Yoshikawa. It follows that the examples given by Cappell and Fox provide a complete list of metabelian 2-knot groups.
\end{abstract}

In [4] we showed that if the commutator subgroup of the group of a 2-knot is finitely generated abelian, then it must be isomorphic to $\mathbf{Z}^{3}$ or finite cyclic of odd order. Yoshikawa [10] has shown that if the commutator subgroup is abelian but not finitely generated then it must be isomorphic to the dyadic rationals $D=\mathbf{Z}\left[\frac{1}{2}\right]$ or to $D \oplus(\mathbf{Z} / 5 \mathbf{Z})$. We shall give a somewhat different argument which shows that only $D$ can occur. To complete the determination of the metabelian 2-knot groups we need to know how the meridians may act by conjugation on the commutator subgroup. In $[4,5]$ it is shown that if the commutator subgroup is $\mathbf{Z}^{3}$ then the meridian action is given by a matrix with positive determinant, while Farber [2] showed that if the commutator subgroup is odd cyclic then the meridian action is inversion. It is easily seen that (up to change of orientation) the only possible meridian action on $D$ is multiplication by 2 . Thus the examples due to Cappell [1] for the case $\mathbf{Z}^{3}$ and to Fox [3] for the cases $\mathbf{Z} /(2 n+1) \mathbf{Z}$ and $D$ exhaust the possible metabelian 2-knot groups.

Let $K: S^{2} \rightarrow S^{4}$ be a 2-knot with group $G=\pi_{1}\left(S^{4} \backslash K\left(S^{2}\right)\right)$, and let $Y$ be the closed 4-manifold obtained by surgery on $K$. (Thus $Y$ has fundamental group $G$.) Suppose that the commutator subgroup $G^{\prime}$ is abelian, but not finitely generated. Then $G^{\prime}$ is a finite extension of $D$, by [6 or 10]. In particular the torsion subgroup $T$ of $G^{\prime}$ is finite, and so $G^{\prime} \approx D \oplus T$ as an abelian group [8, p. 106]. A choice of meridians for $K$ determines an isomorphism $\mathbf{Z}\left[G / G^{\prime}\right] \approx \Lambda=\mathbf{Z}\left[t, t^{-1}\right]$ and hence a $\Lambda$-module structure on $G^{\prime}$. Although $T$ is a submodule of $G^{\prime}$, we do not know a priori whether it is a module direct summand. For $p$ a prime, let $r_{p}$ be the dimension of $T / p T$ as a vectorspace over the prime field $\mathbf{F}_{p}$. We shall use the following injectivity property of cup product together with Milnor duality to estimate these dimensions.

LEMMA. Let $A$ be an abelian group and $F$ a field such that char $F \neq 2$, considered as a trivial $A$-module. Then the map $H^{1}(A ; F) \wedge H^{1}(A ; F) \rightarrow H^{2}(A ; F)$ induced by cup product is injective.

Received by the editors February 11, 1985.

1980 Mathematics Subject Classification. Primary 57Q45.

Key words and phrases. Commutator subgroup, injectivity of cup product, metabelian, Milnor duality, two-knot. 
Proof. The first three cohomology groups for a group $G$ with coefficients in a ring $R$ which is trivial as a $G$-module may be computed as: $H^{0}(G ; R)=R$, $H^{1}(G ; R)=\operatorname{Hom}(G, R)$ and $H^{2}(G ; R)=\left\{F: G^{2} \rightarrow R \mid F(h, j)-F(g h, j)+\right.$ $F(g, h j)-F(g, h)=0$ for all $g, h, j$ in $G\} / B$ where $B=\left\{F_{f}:\langle g, h\rangle \mapsto f(h)-\right.$ $f(g h)+f(g)$ for all $g, h$ in $G \mid$ all functions $f: G \rightarrow R\}$. The cup product of two elements $f_{1}, f_{2}$ in $H^{1}(G ; R)$ is represented by the function sending $(g, h)$ in $G^{2}$ to $f_{1}(g) f_{2}(h)$ in $R$.

There is a natural map $\mu: H^{1}(G ; R) \wedge H^{1}(G ; R) \rightarrow \wedge^{2}\left(G / G^{\prime}, R\right)$ (skew-symmetric bilinear maps from $\left(G / G^{\prime}\right) \times\left(G / G^{\prime}\right)$ to $\left.R\right)$ given by $\mu\left(\sum f_{i} \wedge g_{i}\right)\left(x G^{\prime}, y G^{\prime}\right)=$ $\Sigma\left(f_{i}(x) g_{i}(y)-f_{i}(y) g_{i}(x)\right)$. This map is always injective if $R$ is a field. Now suppose $\sum f_{i} \wedge g_{i}$ in $H^{1}(A ; F) \wedge H^{1}(A ; F)$ is such that $\sum f_{i} \cup g_{i}=0$. Then there is a function $h: A \rightarrow F$ such that $\sum f_{i}(a) g_{i}(b)=h(a)+h(b)-h(a b)$ for all $a, b$ in $A$. Therefore

$$
\begin{aligned}
\mu\left(\sum f_{i} \wedge g_{i}\right)(a, b) & =h(a)+h(b)-h(a b)-(h(b)+h(a)-h(b a)) \\
& =h(b a)-h(a b)=0
\end{aligned}
$$

since $A$ is abelian. By the injectivity of $\mu$, it follows that $\sum f_{i} \wedge g_{i}=0$.

REMARK. In characteristic 2 cup product induces instead a map from the symmetric product $H^{1}(A ; F) \odot H^{1}(A ; F)$ to $H^{2}(A ; F)$. If $F=\mathrm{F}_{2}$ the kernel of cup product is isomorphic to the image of $H^{1}(A ; \mathbf{Z} / 4 \mathbf{Z})$ in $H^{1}\left(A ; \mathbf{F}_{2}\right)$ under reduction modulo 2. For more general groups $G$ the kernel of cup product may be related to the subquotient $G_{2} / G_{3}$ of the lower central series [7,9].

Now let $Y^{\prime}$ be the infinite cyclic covering space of $Y$, so that $\pi_{1}\left(Y^{\prime}\right) \approx G^{\prime}$. If $F$ is a field then $H^{3}\left(Y^{\prime} ; F\right) \approx F$ and cup product: $H^{1}\left(Y^{\prime} ; F\right) \times H^{2}\left(Y^{\prime} ; F\right) \rightarrow H^{3}\left(Y^{\prime} ; F\right)$ is a perfect pairing, by Milnor duality. Moreover $H^{1}\left(G^{\prime} ; F\right)=H^{1}\left(Y^{\prime} ; F\right)$ and $H^{2}\left(G^{\prime} ; F\right) \subseteq H^{2}\left(Y^{\prime} ; F\right)$ (since the natural map $Y^{\prime} \rightarrow K\left(G^{\prime}, 1\right)$ is 2-connected), and so $F$-dim $H^{2}\left(G^{\prime} ; F\right) \leqslant F$-dim $H^{1}\left(G^{\prime} ; F\right)$. If $p$ is an odd prime and $F=\mathbf{F}_{p}$ then $F-\operatorname{dim} H^{1}\left(G^{\prime} ; F\right)=r_{p}+1$ so by the lemma

$$
\left(\begin{array}{c}
r_{p}+1 \\
2
\end{array}\right) \leqslant r_{p}+1
$$

which implies that $r_{p}=0,1$ or 2 . Moreover $r_{p}$ cannot be 1 , for otherwise $F$ $\operatorname{dim} H^{1}\left(G^{\prime} ; F\right)=2$ and the image of $\wedge_{2} H^{1}\left(G^{\prime} ; F\right)$ in $H^{2}\left(Y^{\prime} ; F\right)$ would be 1-dimensional, and so nontrivially paired with another element of $H^{1}\left(G^{\prime} ; F\right)$. But there can be no nonzero alternating trilinear form on a 2-dimensional vectorspace. Therefore $r_{p}=0$ or 2 .

We shall next show that $H_{2}\left(G^{\prime} ; \mathbf{Z}\right)$ must be finite cyclic of odd order. By Hopf's theorem it is a quotient of $H_{2}\left(Y^{\prime} ; \mathbf{Z}\right)$ (even as a $\Lambda$-module) and by equivariant Poincaré duality

$$
H_{2}\left(Y^{\prime} ; \mathbf{Z}\right) \approx \operatorname{Ext}_{\Lambda}^{1}\left(\overline{H_{1}\left(Y^{\prime} ; \mathbf{Z}\right)}, \Lambda\right)=\operatorname{Ext}_{\Lambda}^{1}\left(\overline{G^{\prime}}, \Lambda\right) .
$$

We may assume the meridians so chosen that $G^{\prime} / T \approx \Lambda /(t-2)$ as a $\Lambda$-module, and then $\operatorname{Ext}_{\Lambda}^{1}\left(\overline{G^{\prime}}, \Lambda\right)=\operatorname{Ext}_{\Lambda}^{1}\left(\overline{G^{\prime} / T}, \Lambda\right) \approx \Lambda /(2 t-1)$, which is again isomorphic to $D$ as an abelian group. Now $H_{2}\left(G^{\prime} ; \mathbf{Z}\right) \otimes \mathbf{Q}=H_{2}\left(G^{\prime} ; \mathbf{Q}\right)$ is 0 , as follows from the LHS-spectral sequence for the extension $1 \rightarrow T \rightarrow G^{\prime} \rightarrow D \rightarrow 1$. Therefore 
$H_{2}\left(G^{\prime} ; \mathbf{Z}\right)$ must be cyclic of odd order, being a torsion quotient of $D$. (The argument of this paragraph applies equally well if $G^{\prime}$ is a finite nonabelian extension of $D$.)

Now since $G^{\prime}$ is abelian, $H_{2}\left(G^{\prime} ; \mathbf{Z}\right) \approx G^{\prime} \wedge G^{\prime}$, by [8, p. 334], and so $(D \oplus T) \wedge$ $(D \oplus T)=(D \otimes T) \oplus(T \wedge T)$ is odd cyclic. (This calculation provides another proof that $\mathrm{H}_{2}\left(G^{\prime} ; \mathbf{Z}\right)$ is finite, when $G^{\prime}$ is abelian.) Therefore $D \otimes T$ is odd cyclic and so $r_{p} \leqslant 1$ for all odd $p$. But then we must have $r_{p}=0$ for all odd $p$, and so $T$ is a 2-primary group. Since $T \wedge T$ must also be odd cyclic, it must be trivial and so $T$ is itself cyclic. But since $T$ is a $\Lambda$-submodule of a knot module it must admit an automorphism $t$ such that $t-1$ is also an automorphism, which is impossible if $T$ is cyclic of even order. Therefore $T=0$ and so $G^{\prime} \approx D$.

REMARK. In subsequent work on 2-knot groups [6] the author has shown that if $G$ is solvable and either torsion free or constructible (e.g. if $G^{\prime}$ is nilpotent) then either $G$ is torsion free polycyclic of Hirsch length 4 and orientable type or $G^{\prime}$ is finite (cf. [4]) or $G$ is Fox's group. Moreover if $G^{\prime}=Z^{3}$ then the exterior of the knot is determined up to homeomorphism by $G$ alone.

\section{REFERENCES}

1. S. E. Cappell, Superspinning and knot complements, Topology of Manifolds, Georgia 1969 , Markham, Chicago, Ill., 1970, pp. 358-383.

2. M. S. Farber, Duality in an infinite cyclic covering and even-dimensional knots, Math. USSR Izv. 11 (1977), 749-781.

3. R. H. Fox, A quick trip through knot theory, Topology of 3-Manifolds and Related Topics, Prentice-Hall, Englewood Cliffs, N. J., 1962, pp. 120-167.

4. J. A. Hillman, High dimensional knot groups which are not two-knot groups, Bull. Austral. Math. Soc. 16 (1977), 449-462.

5. __ Orientability, asphericity and two-knot groups, Houston J. Math. 6 (1980), 67-76.

6 . Two-knot groups with torsion free abelian normal subgroups, preprint, Australian National University, 1985.

7. The kernel of the cup product, Bull. Austral. Math. Soc. (1985), in press.

8. D. J. S. Robinson, A course in the theory of groups, Graduate Texts in Math., vol. 80, Springer-Verlag, Berlin and New York, 1982.

9. D. Sullivan, On the intersection ring of compact three-manifolds, Topology 14 (1975), 275-277.

10. K. Yoshikawa, On 2-knot groups with abelian commutator subgroups, Proc. Amer. Math. Soc. 92 (1984), 305-310.

Department of Mathematics, Faculty of Science, Australian National University, GPO Box 4, Canberra ACT 2600, Australia

Current address: Department of Mathematics, Macquarie University, North Ryde, N.S.W. 2113, Australia 